\title{
Kinematic Analysis of a Disk Working Body with a Changing Radius of Curvature
}

\author{
Vladimir I. Konovalov ${ }^{1,}$, Sergey I. Konovalov², and Viktor I. Baradakov ${ }^{3}$ \\ ${ }^{1}$ Chair "Processes and machines in agribusiness" of the faculty of mechanization of Kuban State \\ Agrarian University named after I.T.Trubilin, 350044, Krasnodar, Russia \\ ${ }^{2}$ Experimental farm «Krasnodarskoe» of Kuban State Agrarian University named after I.T.Trubilin, \\ 350044, Krasnodar, Russia \\ ${ }^{3}$ Kuban State Agrarian University named after I.T.Trubilin, 350044, Krasnodar, Russia
}

\begin{abstract}
In recent years, the improvement of the technological process of tillage is based on the use of working bodies with a curved shape of the working surface, which can increase the agrotechnical rates of tillage and reduce its energy intensity. Agricultural implements with disk working bodies are among the most common machines in agriculture. The article presents a kinematic analysis of a disk working body with a changing radius of curvature. Expressions for determining the coordinates, velocity, and acceleration of the arbitrary point on the working surface are obtained.
\end{abstract}

\section{Relevance of research}

With the improvement of crop cultivation technologies, disk working bodies are increasingly being used as the basic working body of a tillage unit [1-4]. This is due to both economic and technological aspects [5-11]. In parallel with the increase in the intensity of the use of disk working bodies, their improvement also occurs [12-14]. If at the turn of the XX century they mainly used flat and spherical disks as universal working bodies suitable for any operating conditions, then in recent years the designs of disk working bodies have significantly expanded their nomenclature and narrowed the scope of their purpose [15-18]. For example, there were coltour disks designed for intensive grinding of crop residues, disk knives or "fleo" disks for working on soils with a large number of crop residues, cone disks, etc. One of the most promising designs is a disk working body with a forming working surface made in a spiral, which allows us to change its curvature, and, consequently, the degree of its impact on the soil.

\section{Problem statement}

The main technological feature of the interaction of the disk working body with the soil is to give it a torque during movement on the working surface. Due to this moment, additional deformations occur in the processed layer, which lead to its crumbling [18-20]. The degree

*Corresponding author: konovalov.v.i@mail.ru 
of crumbling of the treated layer as a result of this interaction depends on a variety of controlled and independent or uncontrolled factors. Unmanageable factors include, first of all, the soil and climatic conditions of work, which to some extent can be predicted and expected to meet the recommended parameters. Among the controlled factors are the design, technological and operational parameters of the working body. These factors determine the degree of interaction of the disk working body with the soil. One of the most characteristic and indicative results of their interaction is the kinematics of the movement of the soil layer. Since the kinematic parameters of the movement of the soil layer are greatly influenced by uncontrolled factors, such as the coefficient of friction, granulometric composition, humidity, etc., it is necessary to introduce a number of assumptions for their study. Such assumptions will include the absence of soil slippage relative to the working surface when it moves with it. In addition, the soil layer is a continuum, and in the process of its movement along the working surface of the disk working body, it does not change its shape, and, consequently, its center of gravity.

\section{Presentation of main research material}

For the kinematic analysis of a disk working body with a changing radius of curvature, we will use the coordinate system (Fig. 1), in which the plane formed by the blade of the disk and representing a circle of radius $R$ with the center at its intersection with the beam $A C$ lies in the $I^{\text {st }}$ octant. Then the radius $A F_{l}$ of this circle will touch the $X O Z$ plane and form an angle $\alpha$ with it, equal to the angle of attack of the disk. At the same time, the radius $A F_{4}$ will form an angle $\beta$ with the same plane, which is equal to the angle of inclination of the disk to the vertical.

From all the points of the forming working surface of the disk working body, the initial position of the point $F$ on the blade of the disk is known, fixed by its radius $R$ and the maximum radius of curvature $\rho_{\max }$, and the position of the point $A$, fixed by the minimum radius $\rho_{\min }$ and angle $\psi_{4}$.

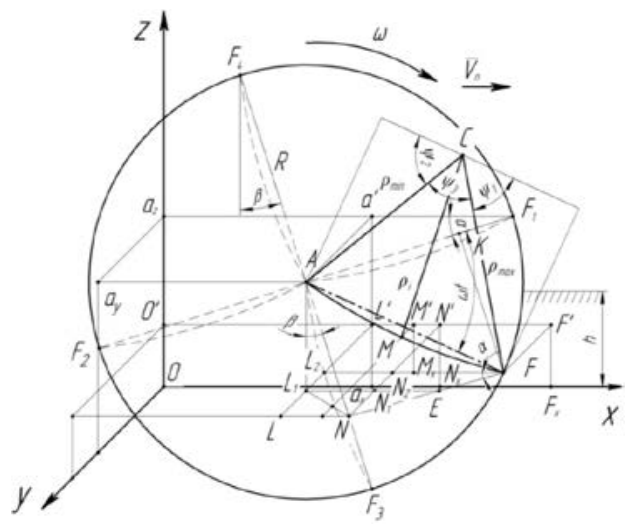

Fig. 1. Scheme for determining the trajectory of an arbitrary point on the working surface of a disk working body with a changing radius of curvature

For the reference point, we take the point $A$ on the working surface of the disk, which will allow us to obtain projections of the arbitrary point $M$ lying in the same octant. Then, in the specified coordinate system, the disk will move in the direction of the $O X$ axis, the point $A$ will initially lie in the YOZ plane, and after a moment of time $t$ will move by an amount equal to $V_{n} t$.

In this case, the trajectory of the arbitrary point $F$ will be described by a system of equations [21]: 


$$
\left\{\begin{array}{l}
x=V_{n} t+R \sin \alpha \sin \beta \sin \omega t+R \cos \alpha \cos \omega t \\
y=R \sin \alpha+R \cos \alpha \sin \beta \sin \omega t-R \sin \alpha \cos \omega t \\
z=R \cos \beta-R \cos \beta \sin \omega t
\end{array}\right.
$$

where $x, y, z$-coordinates of the arbitrary point of the cutting edge of the disk working body; $V_{n}$ - forward speed of the disk working body; $\alpha$ - angle of attack of the disk working body; $\beta$ - angle of inclination of the axis of rotation of the disk working body to the horizon; $\omega$ - angular speed of rotation of the disk working body; $\omega t$ - angle of rotation of the disk working body.

The system of equations (1) will describe the trajectory of the cutting edge of the working body. However, the soil moves along the working surface of the disk at an angle to the radius, and the cutting edge does not participate in this process. Therefore, it is necessary to fix the position of the arbitrary point $M$ on the working surface of the disk working body. Since the point $M$ belongs to the spiral arc bounded by the minimum $\rho_{\min }$ and maximum $\rho_{\max }$ and the radius of curvature, the position of the point $M$ can be fixed using a different value of the angle of rotation $\varphi_{i}$ (Fig. 2) and the corresponding current radius of curvature $\rho_{i}$. In this case, the value of the rotation angle $\varphi_{i}$ will vary from $f_{\min }$ to $\varphi_{\max }$.

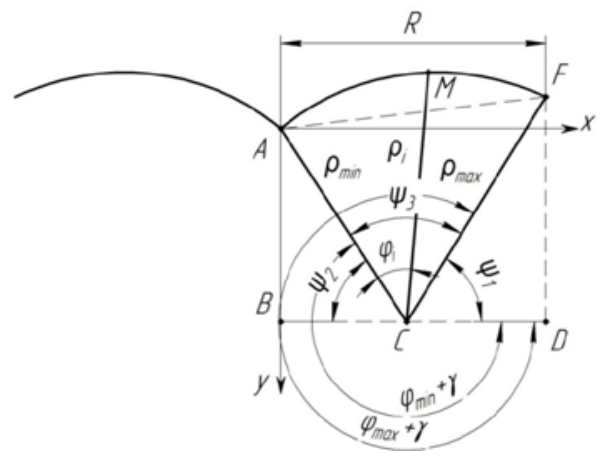

Fig. 2. Scheme for fixing the arbitrary point $M$ of the working surface of a disk working body with the changing radius of curvature

In this case, the fixation of the arbitrary point $M$ of the working surface of the disk working body with the changing radius of curvature will be determined by expressions and symbols:

$$
\begin{gathered}
\rho_{\min } \sin \psi_{2}-k \varphi_{i} \sin \left(\psi_{2}+\varphi_{i}-\varphi_{\min }\right)=A_{1}, \\
\rho_{\min } \cos \psi_{2}-k \varphi_{i} \cos \left(\psi_{2}+\varphi_{i}-\varphi_{\min }\right)=B_{1} .
\end{gathered}
$$

where $\varphi_{i}$ - angle of rotation of the spiral to form the radius of curvature $p_{i}$ of the arbitrary point $M, \varphi_{\min }$ - angle of rotation of the beam of the forming spiral to form a minimum radius of curvature, radius; $\rho_{\max }$ and $\rho_{\min }-$ maximum and minimum radius of curvature, respectively, mm; $\psi_{2}$ - value of the acute angle between the $O X$ axis and minimum radius of curvature.

Then the coordinates of the arbitrary point $M$ will be:

$$
\begin{gathered}
x=V_{n} t+B_{1} \cos \alpha \cos \omega t+B_{1} \sin \alpha \sin \beta \sin \omega t+A_{1} \sin \alpha \cos \beta, \\
y=B_{1} \cos \alpha \sin \beta \sin \omega t-B_{1} \sin \alpha \cos \omega t+A_{1} \cos \alpha \cos \beta+\frac{R \sin \left(\alpha+\psi_{4}\right)}{\cos \psi_{4}}, \\
z=A_{1} \sin \beta-B_{1} \cos \beta \sin \omega t+R \cos \beta
\end{gathered}
$$


After differentiating the coordinates of the same name in time $t$, we obtain the corresponding values of the projections of velocity and acceleration on coordinate axes, which will be described by the expressions:

$$
\begin{gathered}
V_{x}=\frac{d x}{d t}=V_{n}-B_{1} \omega \cos \alpha \sin \omega t+B_{1} \omega \sin \alpha \sin \beta \cos \omega t, \\
V_{y}=\frac{d y}{d t}=B_{1} \omega \cos \alpha \sin \beta \cos \omega t+B_{1} \omega \sin \alpha \sin \omega t \\
V_{z}=\frac{d z}{d t}=-B_{1} \omega \cos \beta \cos \omega t \\
a_{x}=\frac{d^{2} x}{d t^{2}}=-B_{1} \omega^{2} \cos \alpha \cos \omega t-B_{1} \omega^{2} \sin \alpha \sin \beta \sin \omega t \\
a_{y}=\frac{d^{2} y}{d t^{2}}=-B_{1} \omega^{2} \cos \alpha \sin \beta \sin \omega t+B_{1} \omega^{2} \sin \alpha \cos \omega t \\
a_{z}=\frac{d^{2} z}{d t^{2}}=B_{1} \omega^{2} \cos \beta \sin \omega t
\end{gathered}
$$

where $V_{x}, V_{y}$ and $V_{z^{-}}$absolute velocity projections on the coordinate axis; $; a_{x}, a_{y}$ and $a_{z}$ - projections of the absolute acceleration of the arbitrary point $M$ on the coordinate axis.

Using the basic principles of vector algebra, the absolute velocity and acceleration of the arbitrary point $M$ of the working surface of a disk working body with a changing radius of curvature will be described by the expressions [22, 23]:

$$
\begin{gathered}
V_{M}=\sqrt{V_{n}^{2}+B_{1}^{2} \omega^{2}+2 V_{n} B_{1} \omega(\sin \alpha \sin \beta \cos \omega t-\cos \alpha \sin \omega t)} . \\
a_{M}=B_{1} \omega^{2} .
\end{gathered}
$$

where $V_{M}$ - absolute speed of the arbitrary point $M$ of the working surface of the disk working body; $a_{M}$-absolute acceleration of the arbitrary point $M$ of the working surface of the disk working body;

The change in the magnitude and direction of the absolute velocity of the arbitrary point $M$ will characterize the process of discarding the soil formation. To analyze the process of interaction of the processed layer with the working surface of the disk working body, it is necessary to substitute the numerical values of the design and operating parameters into obtained expressions, and build graphical interpretations based on the obtained data. For clarity and brevity of the interpretation of studied parameters, we can use the axonometric projection of the disk divided into 12 parts (Fig. 3-6), and leave the directions of the coordinate and displacement axes of the disk unchanged. In this case, the construction of all projections must be performed at the same scale.

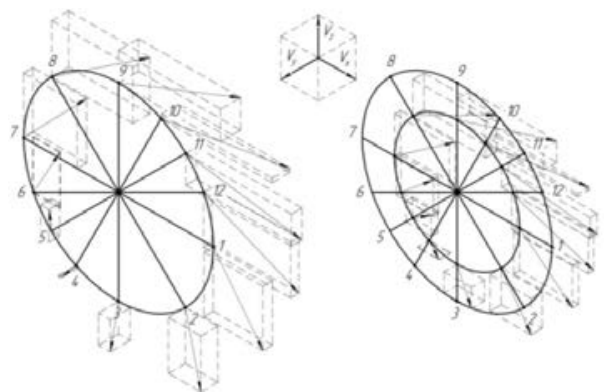

Fig. 3. Diagram of the change in the absolute velocity of the point $M$ of the disk working body with a changing radius of curvature at $V_{n}=3 \mathrm{~m} / \mathrm{s}, \alpha=18^{\circ}, \beta=10^{\circ}, R=0,28 \mathrm{~m}, k=0,095 \mathrm{~m}, \psi_{4}=0^{\circ}$ 


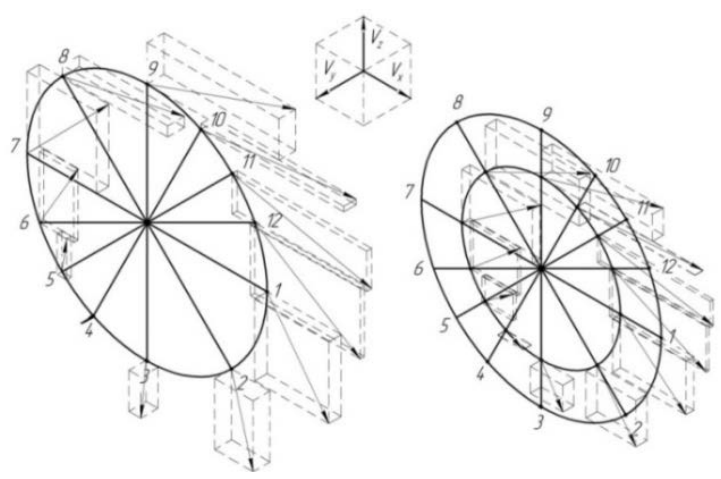

Fig. 4. Diagram of the change in the absolute velocity of the point $M$ of the disk working body with a changing radius of curvature at $V_{n}=3 \mathrm{~m} / \mathrm{s}, \alpha=10^{\circ}, \beta=10^{\circ}, R=0,28 \mathrm{~m}, k=0,095 \mathrm{~m}, \psi_{4}=0^{\circ}$

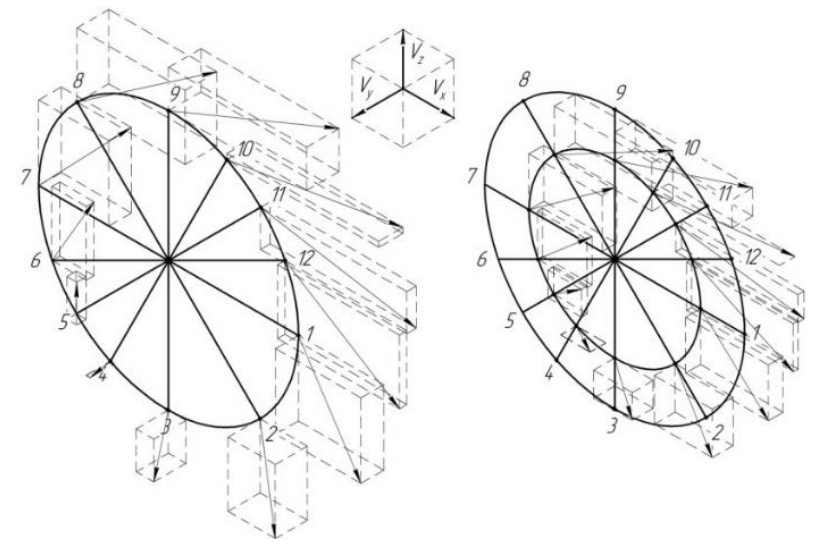

Fig. 5. Diagram of the change in the absolute velocity of the point $M$ of the disk working body with a changing radius of curvature at $V_{n}=3 \mathrm{~m} / \mathrm{s}, \alpha=18^{\circ}, \beta=18^{\circ}, R=0,28 \mathrm{~m}, k=0,095 \mathrm{~m}, \psi_{4}=0^{\circ}$

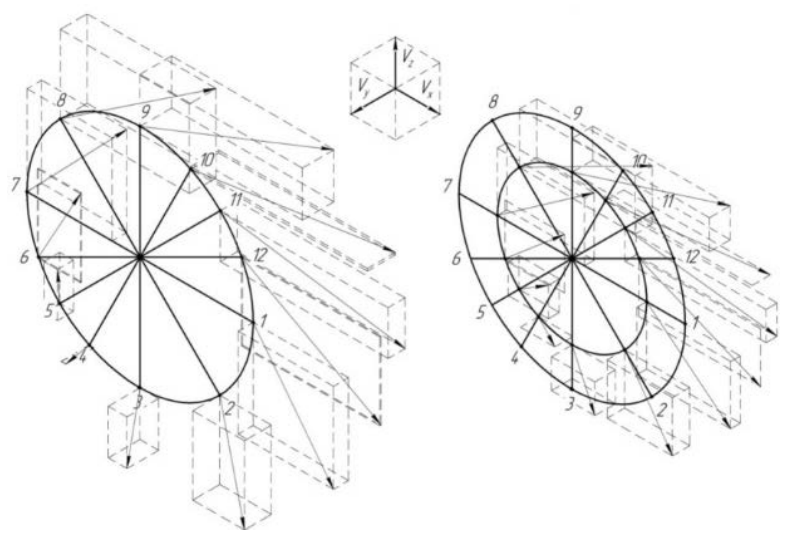

Fig. 6. Diagram of the change in the absolute velocity of the point $M$ of the disk working body with a changing radius of curvature at $V_{n}=4 \mathrm{~m} / \mathrm{s}, \alpha=18^{\circ}, \beta=10^{\circ}, R=0,28 \mathrm{~m}, k=0,095 \mathrm{~m}, \psi_{4}=0^{\circ}$

From obtained diagrams (Fig. 3-6), it can be seen that the absolute velocity of the point $M$ will vary significantly in direction and magnitude. In this case, the minimum value of the speed is at the angle of rotation of the disk $60-120^{\circ}$. The angle between the absolute velocity of the point $M$ at the angle of rotation from 0 to $180^{\circ}$ will be directed from the plane of 
the disk blade towards the unprocessed soil formation, and at the angle from $180^{\circ}$ to $360^{\circ}$ towards the furrow's wall. When turning more than $90^{\circ}$, the absolute velocity vector increases sharply in its magnitude and is oriented vertically, on the basis of which, it can be concluded that the separation of soil formation from the bottom of the furrow and its rise along the working surface will begin after this angle of rotation of the disk.

When analyzing the absolute velocity of the point $M$ located at a distance of $1 / 3$ from the cutting edge, it can be concluded that the general trend of the direction of the velocity vectors in the range of the angle of rotation from 0 to $60^{\circ}$ remains, there is a general decrease in the angle between the absolute velocity vector and the direction of movement, as well as a decrease in the absolute velocity value. When the angle of rotation of the disk is more than $60^{\circ}$, the direction of the velocity vectors, compared with the velocity vectors of the points on the cutting edge, is characterized by a larger value of the components $V_{x}$ and a smaller $V_{z}$, which indicates the occurrence of two differently directed stresses in the soil formation, which will lead to its destruction.

As the angle of attack increases, the absolute velocity vector in the working area increases. The most intense increase in speed occurs in the range of the angle of rotation from 60 to $120^{\circ}$ due to an increase in the components $V_{x}$ and $V y$, while the angle of deviation of the absolute velocity vector from the plane of the blade of the disk increases, which indicates the rejection of the soil at a greater distance. An increase in the angle $\beta$ of the inclination of the disk working body to the vertical leads to a decrease in the absolute velocity vector in the soil discharge zone in the range from 90 to $180^{\circ}$, which reduces the spread of soil and the energy intensity of tillage by reducing the dynamic pressure of the soil formation.

An increase in the translational velocity $V_{n}$ of the disk leads to an increase in the absolute velocity of the disk at any position, while the least intensive growth is observed in the zone of soil layer rejection in the range from 90 to $120^{\circ}$. This will lead to an increase in energy consumption by increasing the compression of the soil within the angle of rotation from 0 to $60^{\circ}$ and increasing the range of its rejection due to greater speed. These facts allow us to conclude that an increase in the velocity will lead to an increase in the degree of soil crumbling not only by creating multidirectional stresses in the soil formation, but also by its fall from a higher height, i.e., giving it greater kinetic energy.

\section{Conclusions}

After conducting a kinematic analysis of the operation of a disk working body with a changing radius of curvature, it can be concluded that the magnitude and direction of the absolute velocity of the arbitrary point are greatly influenced by the progressive $V_{n}$ velocity of the disk and the angle of attack $\alpha$. In addition, in the process of its movement along the working surface of the disk, multidirectional stresses occur in the soil formation, which will increase the degree of its crumbling.

\section{References}

1. S. I. Kambulov, V. P. Maksimov, Yu. A. Tsarev, E. M. Zubrilin, Energy-saving technologies of grain crop cultivation Scientific life, v. 2, pp. 19-26 (2019).

2. E. I. Trubilin, K. A. Sokht, V. I. Konovalov, O. V. Danyukova, Rotary disk working bodies - as a basic element in combined aggregates for soil processing and sowing, Multidisciplinary network electronic scientific journal of Kuban State Agrarian University, v. 91, pp. 465-497 (2013). 
3. E. I. Trubilin, K. A. Sokht, V. I. Konovalov, S. V. Belousov, Disk harrows and huskers in the system of basic and pre-sowing tillage. Problems and ways to solve them, Multidisciplinary network electronic scientific journal of Kuban State Agrarian University, v. 88, pp. 662-671 (2013).

4. G. G. Maslov, V. T. Tkachenko, E. M. Yudina, M. R. Kadyrov, S.A. Kalitko, The improvement of the technology of winter wheat grain production for the purpose of energy saving, Biosciences Biotechnology Research Asia, v. 12(3), pp. 2071-2080 (2015).

5. Yu. K. Kastidi, D. A. Krepyshev, Economic efficiency of the provision with agricultural machinery of commodity producers of Krasnodar Territory, Proceedings of Kuban State Agrarian University, v.28, pp. 39-42 (2011).

6. A. Yu. Nesmiyan. Technical characteristics and agrotechnical performance rates of tillage units, Tractors and agricultural machinery, v. 6, pp.58-64 (2017).

7. A. P. Sokolova, Yu. K. Kastidi, G. F. Bershitskaya, M. E. Trubilin, Influence of provision with machinery on economic rates of plant growing, Agricultural mechanical engineer, v. 2, pp. 22-23 (2015).

8. A. I. Trubilin, Yu. I. Bershitsky, Yu. K. Kastidi, Theoretical aspects of efficiency increase of agricultural machinery use in plant growing, Proceedings of Kuban State Agrarian University, v. 17, pp. 7-11 (2009).

9. G. G. Maslov, E.I. Trubilin, E.V. Truflyak, Parameters optimization for multifunctional aggregates in plant growing mechanization, Research Journal of Pharmaceutical, Biological and Chemical Sciences, v. 7(3), pp. 1919-1926 (2016).

10. G.G. Maslov, E.M. Yudina, A.S. Serguntsov, R.O. Evglevsky, Rational system of multifunctional aggregates for mechanization of plant growing, Research Journal of Pharmaceutical, Biological and Chemical Sciences, v. 9(5), pp. 1177-1185 (2018).

11. V. B. Rykov, S. I. Kambulov, E. I. Trubilin, YU. K. Kastidi, System of energy criteria in the justification of technical units for field production, E3S Web of Conferences, v. 196, p. 01008 (2020).

12. V. I. Khizhnyak, A. Yu. Nesmiyan, V. V. Shchirov, Development of technical units for surface tillage, Bulletin of Agrarian Science of Don, v. S4, pp. 34-39 (2018).

13. G. G. Maslov, E. M. Yudina, D. A. Ushakov, G. E. Samurganov, Modernization of engineering support of crop cultivation, IOP Conference Series: Earth and Environmental Science, v. 624(1), p. 012107 (2021).

14. V. Karpeko, I. Masienko, L. Eranova, Innovation process of machine harvesting of laid peas and its efficiency, E3S Web of Conferences, v. 126, p. 00021 (2019).

15. I. Masienko, A. Vasilenko, L. Eranova, Theoretical study of the forced oscillation effect on subsoil tillage, E3S Web of Conferences, v. 193, p. 01028 (2020).

16. G. G. Maslov, A.V. Palapin, E. M. Yudina, D. A. Ushakov, G. E. Samurganov, The advanced rational process flowsheets for cropping spiked cereals, IOP Conference Series: Earth and Environmental Science, v. 659(1), p. 012038 (2021).

17. A. Serguntsov, V. Serguntsova, N. Malashikhin, Operational parameters and modes of rotary working body for harrowing crops, E3S Web of Conferences. v. 126, p. 00023 (2019).

18. V. Konovalov, S. Konovalov, V. Igumnova, Constructive-technological diagram of the rotary-string cultivator and the definition of its main parameters, E3S Web of Conferences, v. 126, p. 00039 (2019). 
19. V. Konovalov, S. Konovalov, V. Igumnova, Analytical study of the design parameters of the grinding unit of disk harrows, IOP Conference Series: Earth and Environmental Science, v. 403, p. 012086 (2019).

20. A. Serguntsov, V. Serguntsova, Theoretical research of needle-shaped harrow operation expended on soil pinching by needles, E3S Web of Conferences, v. 126, p. 00024 (2019).

21. F.M. Kanarev, Rotary soil-processing machines and tools (Machine engineering, 1983).

22. M. Ya. Vygodsky, Handbook of Higher Mathematics (AST: Astrel, 2006).

23. E. I. Trubilin, V. I. Konovalov, S. I. Konovalov, Theoretical study of ways to increase the efficiency of operation of disk tillage units, Proceedings of Kuban State Agrarian University, v. 65, pp. 165-171 (2017). 